\title{
Analysis of the impact of molecular motions on the efficiency of XL-MS and the distance restraints in hybrid structural biology
}

\author{
Vladimir Svetlov ${ }^{1}$ and Evgeny Nudler*1,2 \\ 1 - Department of Biochemistry and Molecular Pharmacology, New York University School of Medicine, New York, NY 10016, USA; 2 \\ - Howard Hughes Medical Institute, Chevy Chase, MD 20815, USA; * - corresponding author.
}

\begin{abstract}
Covalent cross-link mapping by mass spectrometry (XL-MS) is rapidly becoming the most widely used method of hybrid structural biology. We investigated the impact of incremental variations of cross-linker length have on the depth of XL-MS interrogation of protein-protein complexes, and assessed the role molecular motions in solution play in generation of cross-link-derived distance restraints. Supplementation of a popular NHS-ester cross-linker, DSS, with 2 reagents shorter or longer by $\mathrm{CH} 2-\mathrm{CH} 2$, increased the number of non-reductant crosslinks by $\sim 50 \%$. Molecular dynamics simulations of these cross-linkers revealed 3 individual, partially overlapping ranges of motion, consistent with partially overlapping sets of cross-links formed by each reagent. Similar simulations elucidated protein fold-specific ranges of motions for the reactive and backbone atoms from rigid and flexible target domains. Together these findings create a quantitative framework for generation of cross-linker- and protein fold-specific distance restraints for XL-MS-guided protein-protein docking.
\end{abstract}

Covalent cross-link mapping assisted by mass spectrometry (XL-MS, also CXMS, and CLMS) is a lowresolution method of structural interrogation of proteins and protein-protein complexes (PPCs), which employs chemical probing of reactive groups on protein surfaces with bi-functional covalent cross(X)-linkers ${ }^{1-5}$. Other methods focusing on detection of surface/solvent-accessible amino acids, such as hydrogen-deuterium exchange or photo-oxidation, return information about single residues located either to the surface or the interior of the PPC ${ }^{6,7}$. XL-MS by the virtue of mass spectrometry-assisted identification of covalently X-linked pairs of amino acids provides a set of pairwise distance restraints, dependent on the length of the $X$-linker, the structure and the conformational dynamics of a given PPC. These distance restraints in turn can assist with interpretation of crystallographic and cryo-EM experimental densities, as well as guiding protein folding and docking computations ${ }^{8-12}$.

Lagging behind X-ray crystallography and cryo-EM in resolution and completeness of structural information, XL-MS has a set of distinct advantages over them: it is less sensitive to the purity and homogeneity of the PPC sample, and utilizes broadly distributed instruments, whose high sensitivity accounts for lower sample consumption. Even more importantly, by utilizing cell-permeable X-linkers XL-MS can simultaneously discover the composition and report on the structure of PPCs in vivo, using linear and X-linked peptides, respectively ${ }^{13}$. For a long time since publication of the first experimental application of $\mathrm{XL}^{-\mathrm{MS}^{14}}$ this technique occupied a relatively small niche in structural biology, predominantly populated by proof-ofprinciple publications ${ }^{1}$. The past obstacles to the broad utilization of XL-MS can be traced to the highly laborintensive and idiosyncratic manual validation of the spectral data, the focus on custom-made functionalized reagents, and the relative scarcity and low sensitivity of mass spectrometers ${ }^{1,15,16}$.

Recently XL-MS use has been steadily expanding, propelled by the development of automatic (scoredriven) discovery algorithms ${ }^{17-19}$, and the proliferation of the highly sensitive Orbitrap instruments, greatly facilitating data-dependent data acquisition and thus discrimination between linear and X-linked peptides based on their charge ${ }^{20}$. The portability of the XL-MS pipeline has been further improved by shifting the emphasis to the commercially available amino-reactive NHS-esters, such as disuccinimidyl suberate (DSS) and bis(sulfosuccinimidyl)suberate (BS3) ${ }^{21}$. These reagents offer numerous advantages in addition to their broad availability: their chemical properties and reactivity are studied in far greater detail than the rest of X-linker reagents; at near neutral $\mathrm{pH}(6.5-8.0)$ NHS-esters react predominantly with Lys residues (and the N-terminal 
amines), allowing for the formulation of robust discovery rules ${ }^{21-25}$; the relatively high abundance of Lys residues and their preferential localization to protein surfaces ( 6 and $99 \%$, respectively) ${ }^{26,27}$ provides for the high abundance of X-links; X-linker aliphatic spacer doesn't excessively fragment during sample preparation or processing (thereby reducing sample loss and improving discovery rate) ${ }^{1,28-31}$.

The biggest remaining obstacle to the realization of XL-MS full potential as a hybrid structural biology methodology lies in the lack of the detailed understanding of the origins and the nature of the distance restraints it produces. The original idea of the covalent X-link serving as a Euclidean "molecular ruler"28 connecting two residues has been transformed into the one of a X-linker acting as a upper-limited proximity sensor, yet the exact boundaries of this proximity are still poorly elucidated ${ }^{32}$. In this work we report a largescale XL-MS interrogation of a multi-subunit, high-value target PPC (E. coli RNA polymerase ${ }^{33}$ (RNAP)) with 3 different $X$-link reagents of incrementally varied length, interpreted in the framework of molecular dynamics (MD) simulations of the relevant distance restraints ${ }^{34}$. The report concludes with recommendations on incorporating XL-MS-derived distance restraints into PPC docking applications and improving the depth of XLMS experiments by combining X-linkers of different length.

\section{Results}

Two main approaches exist for determining the boundary of distance restraints set by covalent X-links. The first, geometric approach defines the upper limit of such boundary as the Euclidean length of the fully extended X-link; in case of Lys-Lys X-links this is calculated as NZ(=N $\zeta)-N Z, C B\left(=C_{\beta}\right)-C B$, or $C A\left(=C_{\alpha}\right)$-CA distance (Fig. 1A). The second approach derives distance restraints from empirical XL-MS data by measuring distances between X-linked residues in experimental (X-ray, and cryo-EM) protein structures ${ }^{35,36}$. In order to inject physical realism into determination of such measurements, Aebersold and colleagues introduced a nonEuclidean Solvent-Assessible Surface Distance (SASD) ${ }^{37}$. Unfortunately, in XL-MS experiments a fraction of Xlinks is routinely scored as "aberrant" due their apparent length exceeding that of Euclidean upper limit. Moreover, shorter X-linkers tend to produce 2-4 times fewer X-links than the longer ones, although the average length of the X-links in both groups differ only marginally ${ }^{1}$. The uncertainty regarding the "real" length of the experimental X-links and the chances of poisoning the dataset with "aberrant" ones continue to impede successful application of XL-MS in structural biology. X-link-guided protein-protein docking is particularly vulnerable to the uncertainty of the distance restraints. Published reports vary in the estimates of the impact XL-MS-derived restraints had on docking efficiency, and whether SASD performed better than the simple Euclidean distance $e^{9,29,32,35,38,39}$.

We posit that, once $X$-link discovery protocol has been validated through structural benchmarking and manual inspection of the spectra, it does not produce any "aberrant" $X$-links; instead all the high-confidence Xlinks report on the state of the target PPC in solution. The source of the "aberrant" X-links can be then traced to the aberrant states of the PPC, namely to the presence of misfolded PPC components (deviations from the monomer structure), and the oligomerization of the PPC itself (deviations from the oligomer structure). Although non-specific aggregation of proteins in solution does not produce high-abundance reproducible $X$ links (albeit it does reduce the overall discovery rate), specific aggregation (dimerization, etc) can not only reduce the prominence of structure-conforming X-links, but also introduce an oligomer-specific X-linked pairs which would be inadvertently scored as "aberrant" in the monomer structure. The aggregation of the sample in XL-MS experiments can be monitored by dynamic light scattering $(D L S)^{40}$, both before and during X-linking, and its negative impact minimized by the optimization of the experimental conditions and/or removal of the aggregated sample from the pipeline.

Derivation of the distance restraints from the length of the X-linker or based on the location of the Xlinked residues in the static crystallographic or cryo-EM models fails to account for the conformational flexibility of the X-linker and mobility of the PPC in solution. Thermal molecular motions, largely suppressed in X-ray crystallography and cryo-EM, effectively convert both types of molecules into variously populated 
ensembles, thereby introducing uncertainty into distance restraints. First evaluation of the conformational flexibility of X-linkers assessed by MD simulations was provided by Houk and colleagues, indicating that DSS in solution populates a number of states, significantly compacted relative to its extended conformation ${ }^{41}$.

Unfortunately, this work has not been expanded much further. Conformational mobility of the PPCs has even greater impact on the derivation of the distance restraints. It can be divided into two types of motion: thermal and concerted. The first type comprises motions of the Lys side chains relative to the backbone $\left(N Z_{i}-C A_{i}\right.$ vector), motions of the backbone within given domain $\left(C \mathrm{~A}_{i}-\mathrm{CA}_{\mathrm{j}}\right)$, and motions of the domains relative to each other. These can be assessed by subjecting an atomic resolution structure of PPC to MD simulations. The second type of motion comprises non-random, large-scale, and well-defined movements of domains relative to each other as a result of binding to ligands, enzyme's mechano-chemical cycling, etc ${ }^{42,43}$. Such concerted motions can not be fully explored by the MD simulations, without the input of information about the starting and final conformations, and are outside the scope of this work.

In order to explore molecular motions of covalent X-linkers and the residues they react with we have subjected aliquots of a highly monodisperse, monomodal ( $8.5 \%$ polydispersity, $98 \%$ of mass estimated as $\sim 385$ $\mathrm{kDa}$ (vs theoretical mass of $389 \mathrm{kDa}$ )) preparation of E. coli RNAP to X-linking with a panel of reagents, comprised of Adipic acid bis[N-hydroxysuccinimide ester] (DSM4, featuring 4 methylene groups in its spacer), DSS (DSM6), and Disuccinimidyl sebacate (DSM8) (Fig. 1B-D). XL-MS data for each X-linker was filtered by false discovery rate $(<5 \%)$ and e-value $(\leq 0.001)^{44}$, and pooled from several individual experiments to yield 3 datasets containing 5000 individual spectra/precursors (5021, 5130, and 5156, respectively) (Supplementary Table 1).

\section{Combining X-linkers of different length dramatically improve the coverage of XL-MS dataset. RNAP} contains 202 Lys residues, 197 (97.5\%) of which were discovered to form X-links with one or more X-linkers. All 3 Lys present in the smallest RNAP subunit, RpoZ, formed all 3 types of X-links, whereas the larger subunits exhibited minor differences in reactivity: out of 16 Lys residues present in RpoA 12 were found in peptides Xlinked by all 3 reagents, 1 was found in DSM6- and DSM8-derived data, 1 - only among DSM4 X-links, 1 - only among DSM8 X-links, and 1 was not discovered in any of the X-linked peptides. Similarly, 85 out total 87 Lys residues present in the largest subunit, $\mathrm{RpoC}$, were found in the X-linked peptides, 75 formed X-links of all 3 types, 2 were found only in DSM4 dataset, 1 - in DSM4 and DSM8 datasets, 5 - in DSM6 and DSM8 datasets, and 2 - in DSM8 dataset only. Altogether the residues reacting only with subsets of X-linkers are responsible for less than $1 \%$ of total $X$-links and therefore can not explain the 2-3 fold difference in the number of X-links formed by shorter and longer X-linkers ${ }^{1}$.

Analysis of the abundance (the number of precursors per each unique X-link) of X-links distributed among the 3 datasets revealed that the shortest X-linker, DSM4, tends to form a few highly abundant X-links, whereas the majority of $X$-links have a small (less than $0.1 \%$ of the total) number of precursors, drastically reducing the chances of their discovery in each individual experiment (usually $\leq 1000$ precursors). 783 DSM4derived X-links have a total of 5021 corresponding precursors, with 3 most abundant ones represented by 318 , 254, and 131 precursors (14\%), and top 20 X-links - by 1477 precursors (29.4\%). In comparison, abundance of $\mathrm{X}$-links generated by DSM6 and DSM8 is distributed more evenly: the top $3 \mathrm{X}$-links in each case account for $115 / 98 / 83$ and 93/65/65 precursors, respectively, with top 20 being represented by $19.7 \%$ (DSM6) and $18.2 \%$ (DSM8) of total number of precursors. Since in each individual XL-MS experiments the data file contains 500$1000 \mathrm{X}$-linked precursors, and at least 1 precursor is required for X-link discovery, the highly uneven sampling by DSM4 is likely to result in a lower number of unique X-links, compared to that of longer X-linkers, DSM6 and DSM8.

Together the three X-linkers lead to discovery of 1315 non-redundant X-links, including 481 inter-subunt ones (Fig. 2). DSM4 accounted for 783 total X-links, 167 of them not found in DSM6- and DSM8-derived datasets. Similarly, DSM8 experiments yielded $793 \mathrm{X}$-links, among which 121 were unique to this reagent. The most commonly used X-linker, DSM6 (DSS) accounted for 907 X-links, 210 of them being DSM6-specific. In terms of the depth of coverage the use of DSM4 and DSM8 improves the XL-MS discovery rate by $44.9 \%$, compared to DSM6 alone. This increase is greater than those reported in the literature, and does not require switching to a different X-linker chemistry or altering the conditions of X-linking and/or sample processing ${ }^{29}$. 


\section{X-linkers exhibit significant conformational flexibility in solution depending on the length of the}

spacer. The extended Euclidean length of the X-linker provides the theoretical upper limit of the X-link distance restraint, but since the length of the longer X-link fully encompasses that of the shorter one, it fails to account for the unique $X$-links formed by the latter. Following the example of Houk and colleagues and our own MD simulation of $\mathrm{BS}^{45}$, we have carried out 25 ns-long full-atom MD simulations of DSM4, DSM6, and DSS8 in explicit water $^{41}$. The spacer length was recorded as [C4;C9] (DSM4), [C4; C11] (DSM6), and [C4,C13] (DSM8) (Supplementary Tables 2-4). Consistent with the previous reports and the notion of X-linkers conformational flexibility, these simulations revealed that in solution $X$-linkers adopt a range of conformations shorter than their Euclidean length (Fig. 3). DSM4 linker average length is $5.8 \AA$ vs extended $6.3 \AA$, ranging from 3.6 to $6.6 \AA$. DSM6 range of conformations, 4.1-9.1 $\AA$, overlaps with that of DSM4, its average length being $7.7 \AA$ compared to the Euclidean one of $8.7 \AA$. Similarly, the longest X-linker in the series, DSM8, has an average length of $9.9 \AA$, down from the Euclidean of $11.2 \AA$. Its range of 5.5-11.7 $\AA$ partially overlaps not only with that of DSM6, but also of DSM4. This partial overlap is consistent with a subset of X-links common to all $3 \mathrm{X}$-linkers, as well as with presence of unique, $X$-linker-specific $X$-links in each dataset. In addition, these simulations provide first principles, quantitative estimates of the distance restraints (lower and upper limits, as well as the median ("target") distance) imposed by the X-linkers for use in X-link-guided protein-protein docking.

The distance between $X$-linkable amino acids in solution is determined by the fold of the protein and the thermal motions within the protein domains. Conformational dynamics is often invoked in treatment of $X$ links as distance restraints, but it has not been addressed explicitly and/or quantitatively. Instead, in order to account for this uncertainty in data, the upper limit of X-link restraints has been arbitrarily extended above its Euclidean length. Such indiscriminate, "one-size-fits-all" relaxation of the XL-MS-derived distance restraints is the likely reason why in some cases these restraints failed to significantly improve the quality of the docking models. We have reasoned that the conformational mobility of the X-link-reactive residues is affected by the type of the protein fold they are embedded in, thereby allowing assigning fold-specific restraints values to the individual $\mathrm{X}$-links in the experimental data ${ }^{46}$.

To contrast the fold-dependent motions within protein domains we have extracted the atomic coordinates of 2 domains from the structure of E. coli RNAP (4lk1 (Fig. 4A)): a rigid $\alpha$-helical hairpin (RpoB residues 936-1046 (Fig. 4B)) and a flexible loop domain (RpoC residues 36-103 (Fig. 4C)), and subjected them to the full-atom, explicit water MD simulations as described above. The distances between the actual Xlinkable amines $\left(N Z_{i}-N Z_{j}\right)$ and the backbone carbon atoms $\left(\mathrm{CA}_{\mathrm{i}}-\mathrm{CA}_{\mathrm{j}}\right)$ were recorded over the course of $20 \mathrm{~ns}$ simulations (Supplementary Tables 5 and 6). $C A_{i}-C B_{i}$ were also recorded and showed $<0.15 \AA$ variation (Supplementary Table 5), arguing that behavior of $C B_{i}-C B_{j}$ pairs is essentially congruent to that of $C A_{i}-C A_{j}$ ones. Consistent with the general expectations residues in the unstructured flexible loop exhibited broader range of motions than those from the $\alpha$-helical hairpin, with NZ-NZ atoms showing larger displacement relative to the starting structure than CA-CA pairs. Residues forming experimental intra-loop X-links, RpoC50-RpoC66 and RpoC50-RpoC87, started with NZ-NZ distances of 10.0 and 8.9 $\AA$, respectively, but in the course of the MD simulation reached minimum distances of 4.7 and $5.2 \AA$, and maximum distances of 32.3 and $25.3 \AA$, with median values of 18.7 and $13.4 \AA$, respectively (Fig. 5A). CA-CA trajectories for the same pairs of X-linkable residues exhibited lower levels of displacement from the starting distances of 10.8 and $14.8 \AA$, respectively, to the minimum of 9.0 and $10.3 \AA$ and maximum of 27.2 and $22.3 \AA$ (Fig. 5A). The median distances were 16.3 and $15.2 \AA$. The range of motions for residues embedded in the $\alpha$-helical hairpin found among experimental $X$ links were as follows (Fig. 5B). CA-CA distances for RpoB941-RpoB1035 and RpoB988-RpoB1007 at the start of the simulations were 14.9 and $19.7 \AA$, respectively. Over $20 \mathrm{~ns}$ of simulation time they reached minimum values of 14.1 and $16.3 \AA$, maximum values of 24.3 and $20.6 \AA$, with respective median distances of 17.0 and $17.9 \AA$. NZ-NZ distance variations were larger than those of CA-CA within the same domain, but less dramatic compared to NZ-NZ displacements in the unstructured loop: min-to-max displacements of 9.9 and $13.4 \AA$ (RpoB988-RpoB1007 and RpoB941-RpoB1035) vs 20.1 and 27.6̊̊ (RpoC50-RpoC87 and RpoC50-RpoC66) (Fig. 6A). This trend extends to the displacement of $C A$ atoms in the rigid and flexible domains, notably of lower amplitude compared to that of NZ ones (Fig. 6B). These data provide quantitative computational framework 
for the explicit treatment of XL-MS-derived distance restraints in docking simulations and other hybrid methods of structural biology $10,11,35,39,46,47$. They allow to transition from an indiscriminate single distance restraint ${ }^{28}$ to a set of protein/domain-specific ones, and support the notion that the use of CA-CA restraints reduces the uncertainty arising due to molecular thermal motion, as compared to the more intuitive NZ-NZ restraints.

\section{Discussion}

Our extensive experimental and computational analysis of XL-MS methodology applied to structural interrogation of a multi-subunit bacterial RNAP core provided a number of important insights which can lead to further improvement and broader application of this approach. We have reported a $44.9 \%$ increase in the depth of XL-MS analysis of the already highly efficient NHS-ester-based X-linking by introducing 2 additional reagents (DSM4 and DSSeb(DSM8) in additional to the widely used DSS (DSM6). The addition (DSM8) or removal (DSM4) of 2 methylene groups from the X-linker spacer (relative to DSS), responsible for this increase, did not require modifications to the experimental conditions or sample processing (except for the straightforward alterations of X-link search parameters). It bears noting that DSM4 and DSM8 are now either commercially available, or can be economically made to order at a proteomics grade, allowing for immediate and broad deployment of this approach.

This depth of X-linking was achieved without any enrichment for X-linked peptides, apart from the datadependent selection of ions charged at $\geq 4$ during LC-MS/MS. Incorporation of the orthogonal methods of enrichment, such as the use of multiple proteases or the off-line fractionation by strong cation exchange and size exclusion peptide chromatography, would lead to further improvements of depth of coverage and X-link discovery rates ${ }^{48-50}$, especially for high complexity or low abundance in vivo samples.

The quantitative exploration of DSM4, DSM6, and DSM8 conformational mobility in MD simulations revealed the basis of the substantial overlap in the sets of non-redundant X-links formed by each reagent, and the generation of $X$-links unique to each set. Atomic trajectories in each simulation also allowed us to offer quantitative estimates of the X-linker-specific distance restraints (their median lengths, as well as the upper and lower limits) to replace currently underperforming estimates, based solely on the Euclidean length of the X-linker. Using extensive datasets ( 5000 precursors each) of high-confidence X-links we have discovered that the shorter X-linker generated data skewed towards a few extremely abundant X-links, thereby reducing the overall number of non-redundant entries in each individual experiment. This finding is particularly important for XL-MS interrogation of high-value/low-abundance samples. Whenever the sample scarcity precludes an extensive optimization of the experimental conditions, longer X-links are more likely to produce a greater number of non-redundant X-links than the shorter ones (DSM8>DSM6>DSM4) (VS and EN, unpublished observations).

Explicit MD simulations of thermal motions of the experimentally $X$-linked residues provided realistic, quantitative estimates of the fold-dependent distance restraints. We were able to demonstrate that residues embedded into the flexible, unstructured loop regions undergo motions, vastly exceeding those of residues from the more compact domains. Together these findings indicate that, in order to improve the quality of Xlinks-guided protein-protein docking, the distance restraints involving residues from the flexible/highly mobile domains should be given less weight/lower violation penalties compared to the ones connecting residues from rigid domains. They also argue that use of CA-CA restraints substantially reduces the uncertainty in the data (thereby improving docking efficiency) compared to more relaxed NZ-NZ restraints (see Supplementary Discussion for more details).

\section{Acknowledgements}

We would like to thank Zixuan Li for technical assistance and Tom Waltz for critical reading of the manuscript and invaluable insights. This work was supported by the NIH grant R01 GM107329 and by the Howard Hughes Medical Institute. 


\section{Competing interests}

None declared.

\section{Contributions}

EN and VS conceived the study. VS carried out the experiments and data analysis under EN supervision. EN and VS wrote the manuscript.

\section{Materials and Correspondence}

All correspondence and requests for materials should be submitted to Evgeny Nudler (Evgeny.Nudler@nyumc.org) 
Figure $1 A$

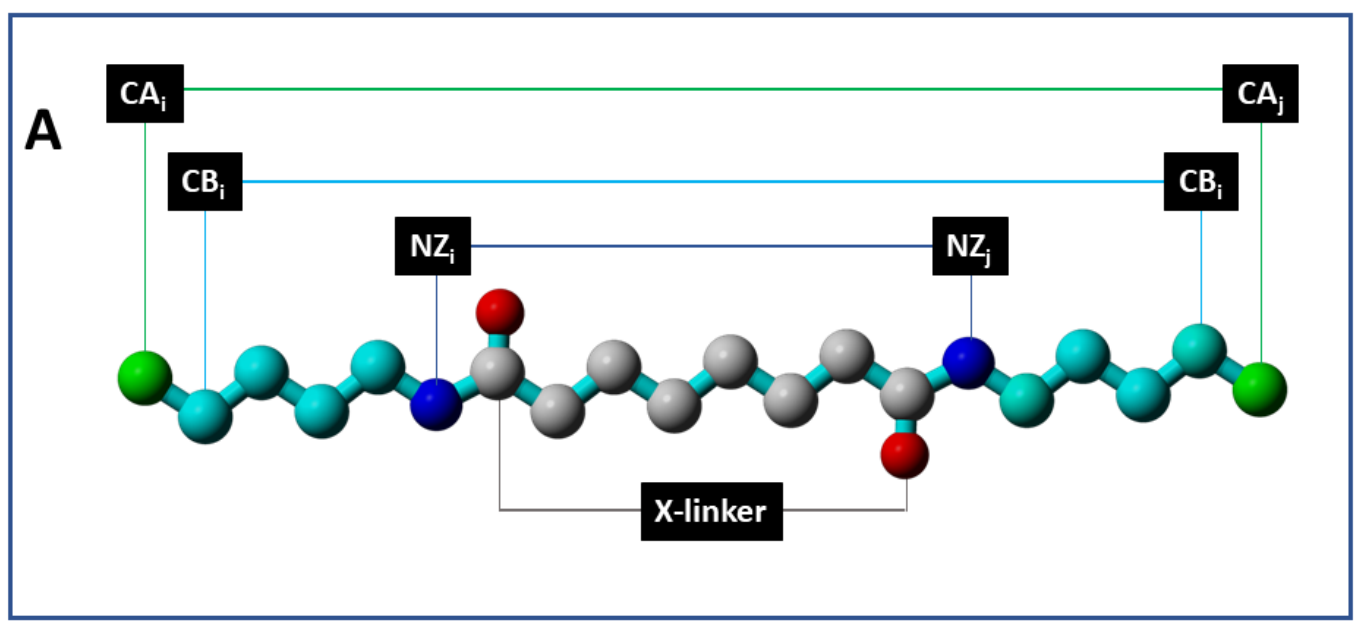

Figure 1B-D
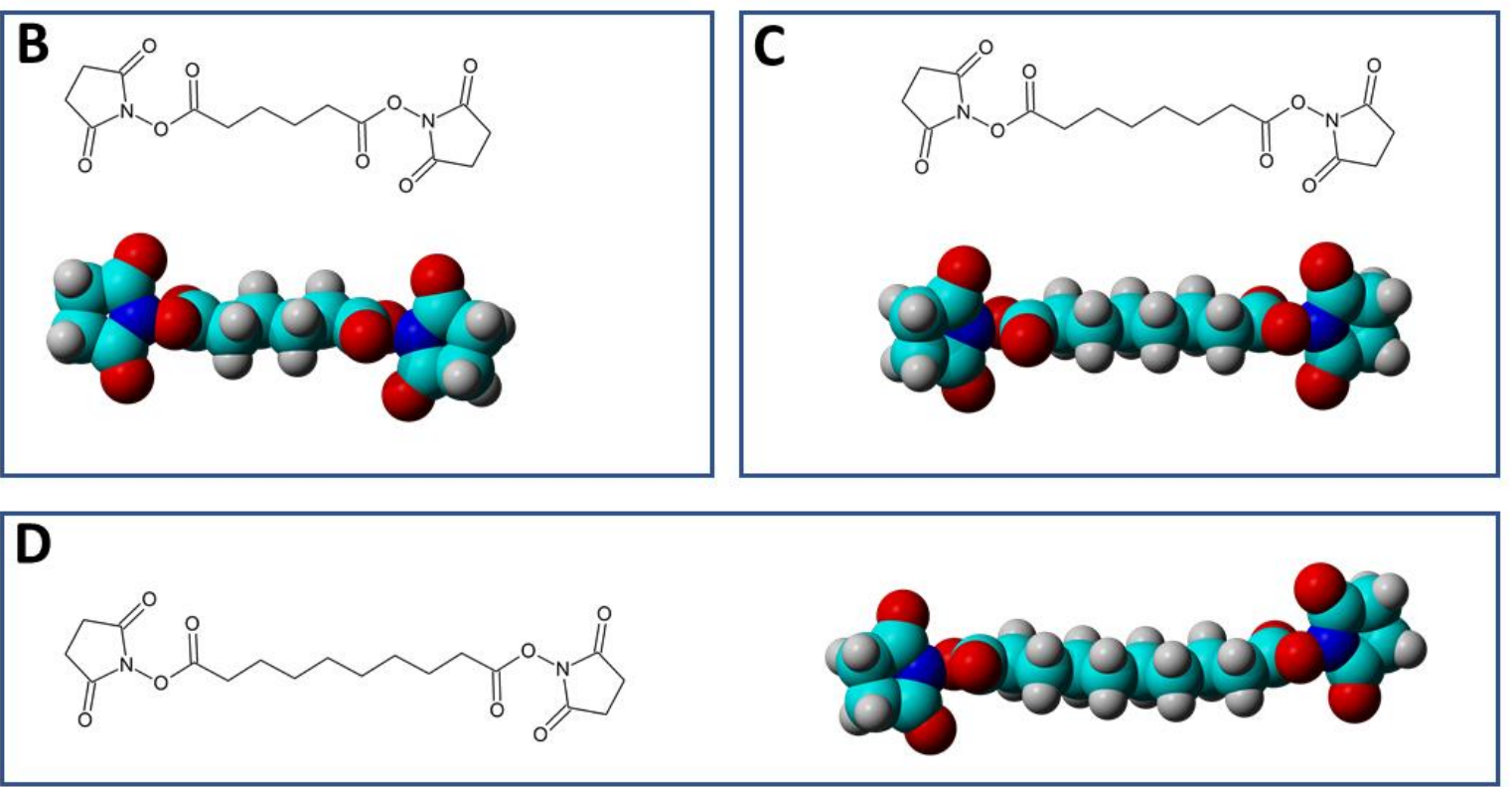
bioRxiv preprint doi: https://doi.org/10.1101/379289; this version posted July 29, 2018. The copyright holder for this preprint (which was not certified by peer review) is the author/funder. All rights reserved. No reuse allowed without permission.

\section{Figure 2}

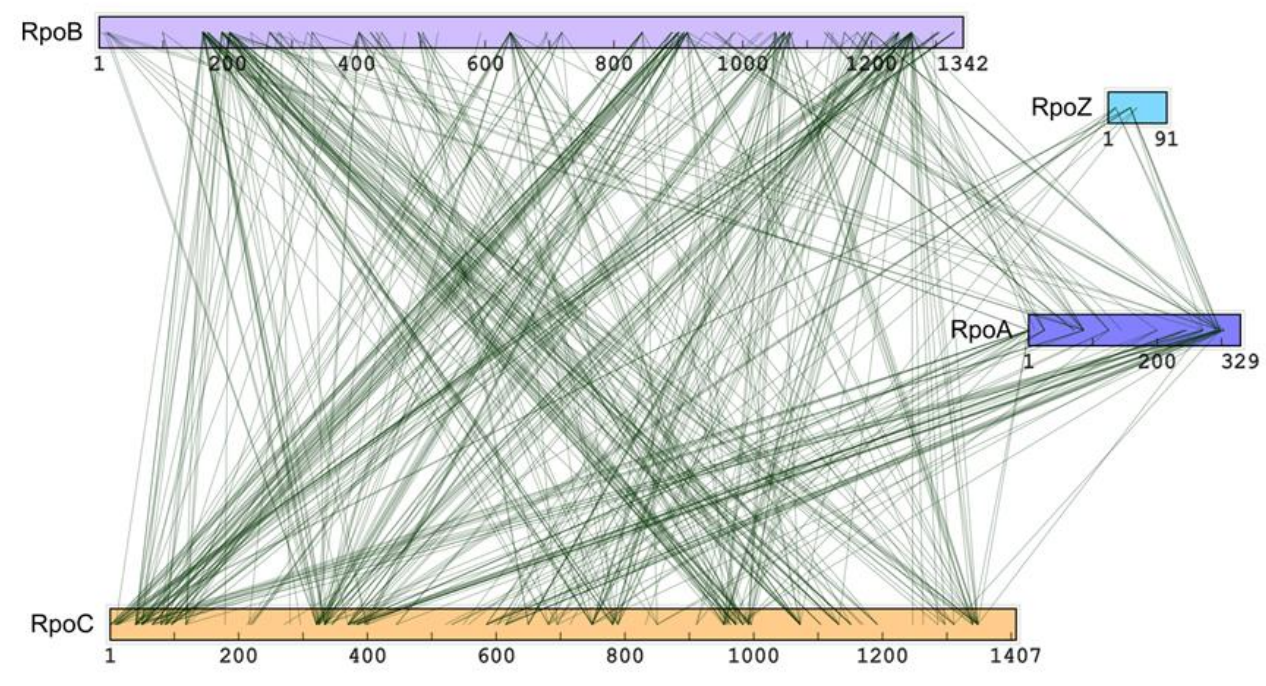


bioRxiv preprint doi: https://doi.org/10.1101/379289; this version posted July 29, 2018. The copyright holder for this preprint (which was not certified by peer review) is the author/funder. All rights reserved. No reuse allowed without permission.

\section{Figure 3}

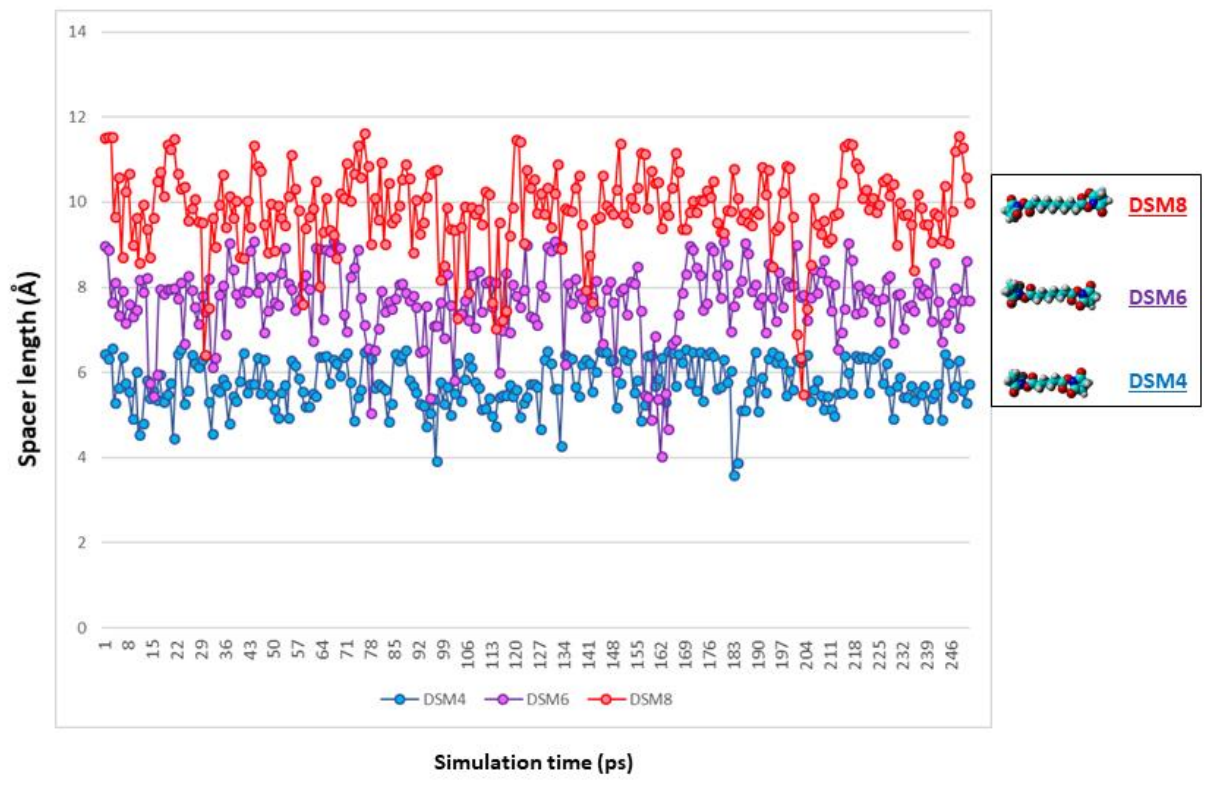


bioRxiv preprint doa: https://doi.org/10.1101/379289; this version posted July 29, 2018. The copyright holder for this preprint (which was not certified by peer review) is the author/funder. All rights reserved. No reuse allowed without permission.

Figure 4A

A

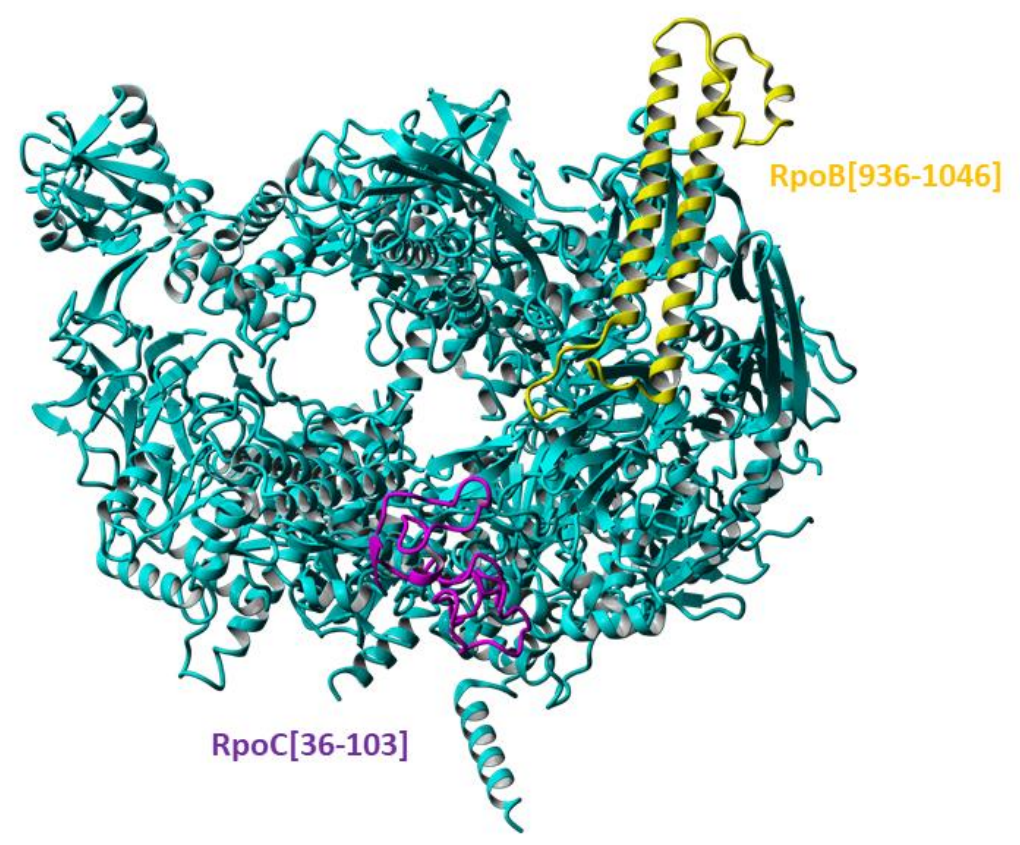

Figure 4B

B

RpoB941

C

RpoB1035

RpoB988

RpoB1007 
bioRxiv preprint doi: https://doi.org/10.1101/379289; this version posted July 29, 2018. The copyright holder for this preprint (which was not certified by peer review) is the author/funder. All rights reserved. No reuse allowed without permission.

\section{Figure 4C}

C

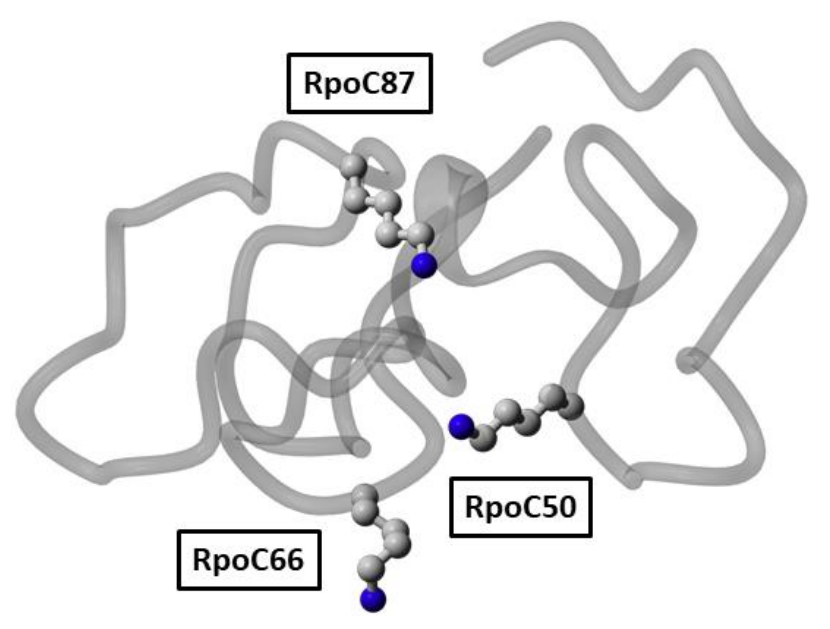


bioRxiv preprint doi: https://doi.org/10.1101/379289; this version posted July 29, 2018. The copyright holder for this preprint (which was not certified by peer review) is the author/funder. All rights reserved. No reuse allowed without permission.

\section{Figure 5A}

A

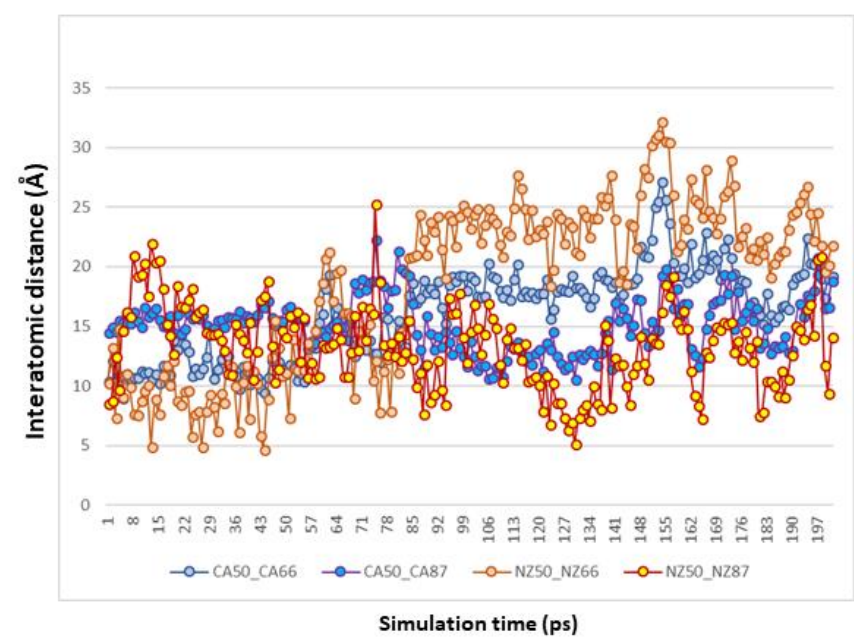

Figure 5B

B

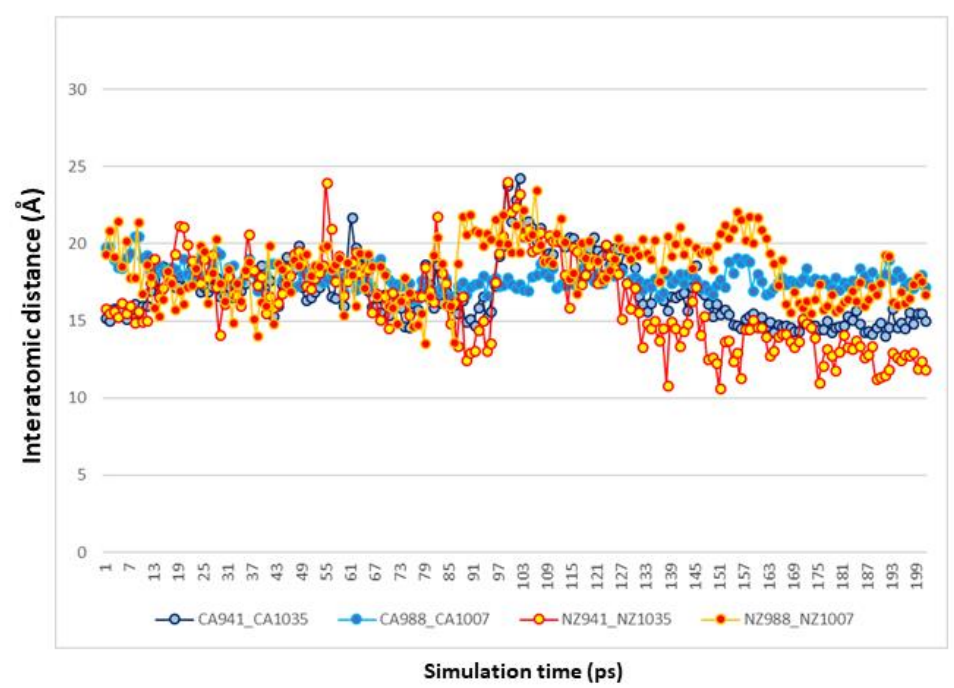


bioRxiv preprint doi: https://doi.org/10.1101/379289; this version posted July 29, 2018. The copyright holder for this preprint (which was not certified by peer review) is the author/funder. All rights reserved. No reuse allowed without permission.

Figure $6 A$

A

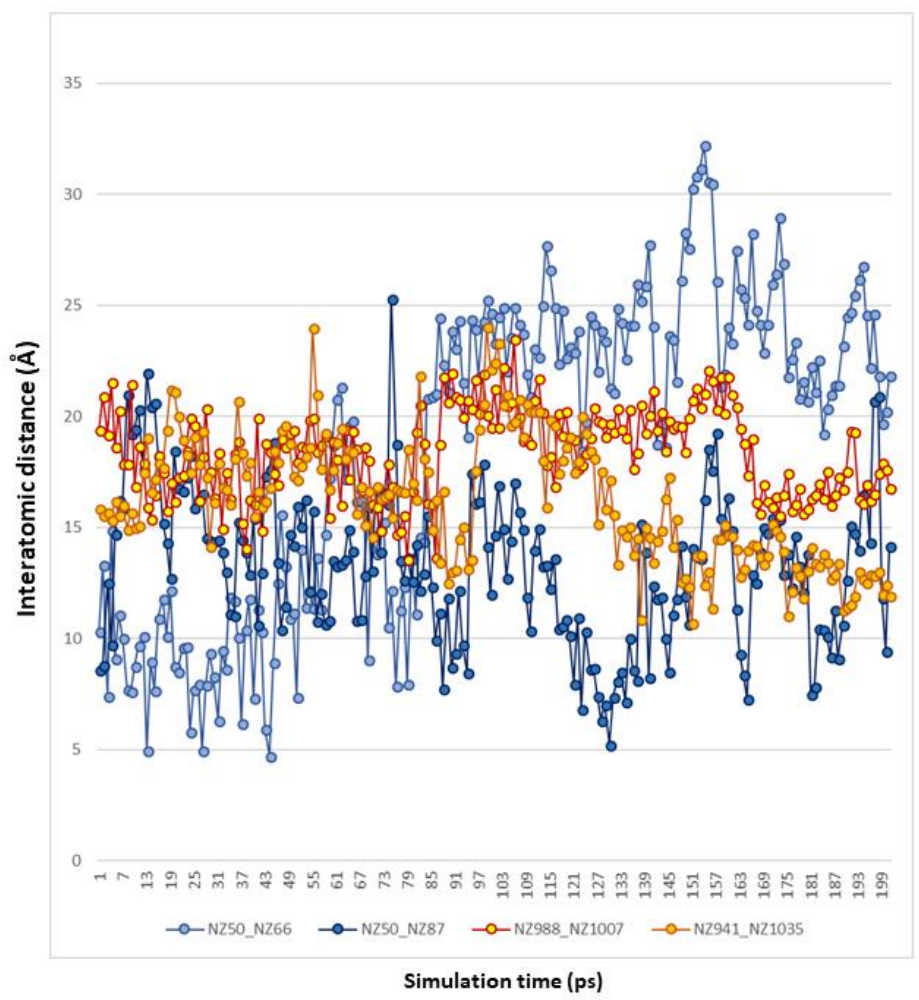

Figure 6B

B

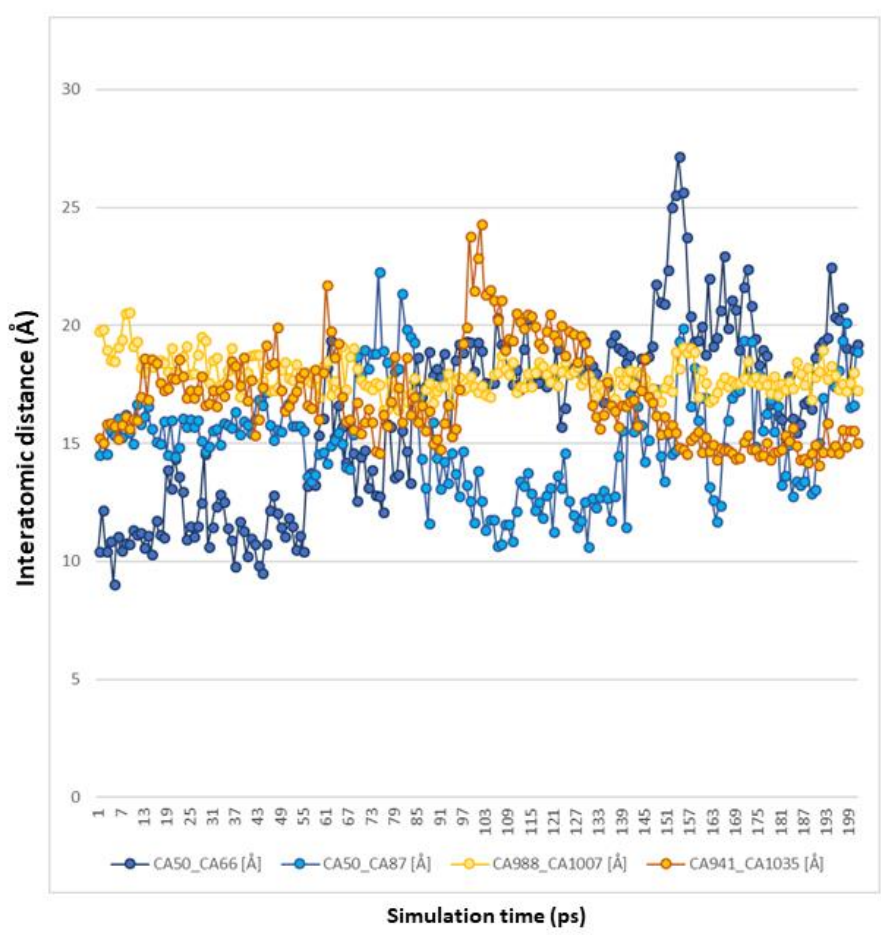




\section{References:}

1 Leitner, A. et al. Probing native protein structures by chemical cross-linking, mass spectrometry, and bioinformatics. Mol Cell Proteomics 9, 1634-1649, doi:10.1074/mcp.R000001-MCP201 (2010).

2 Sinz, A. The advancement of chemical cross-linking and mass spectrometry for structural proteomics: from single proteins to protein interaction networks. Expert Rev Proteomics 11, 733-743, doi:10.1586/14789450.2014.960852 (2014).

Schneider, M., Belsom, A. \& Rappsilber, J. Protein Tertiary Structure by Crosslinking/Mass Spectrometry. Trends Biochem Sci 43, 157-169, doi:10.1016/j.tibs.2017.12.006 (2018). networks by cross-linking mass spectrometry. Curr Opin Struct Biol 35, 100-108, doi:10.1016/j.sbi.2015.10.006 (2015). Aebersold, R. \& Mann, M. Mass-spectrometric exploration of proteome structure and function. Nature 537, 347-355, doi:10.1038/nature19949 (2016). Marcoux, J. \& Cianferani, S. Towards integrative structural mass spectrometry: Benefits from hybrid approaches. Methods 89, 4-12, doi:10.1016/j.ymeth.2015.05.024 (2015). Artigues, A. et al. Protein Structural Analysis via Mass Spectrometry-Based Proteomics. Adv Exp Med Biol 919, 397-431, doi:10.1007/978-3-319-41448-5_19 (2016). Technology to Understand the Structure and Function of Molecular Machines. Trends Biochem Sci 41, 20-32, doi:10.1016/j.tibs.2015.10.008 (2016). Struct Biol 28, 96-104, doi:10.1016/j.sbi.2014.08.001 (2014). Karaca, E., Melquiond, A. S., de Vries, S. J., Kastritis, P. L. \& Bonvin, A. M. Building macromolecular assemblies by information-driven docking: introducing the HADDOCK multibody docking server. Mol Cell Proteomics 9, 1784-1794, doi:10.1074/mcp.M000051-MCP201 (2010).

11 Kahraman, A. et al. Cross-link guided molecular modeling with ROSETTA. PLoS One 8, e73411, doi:10.1371/journal.pone.0073411 (2013).

12 Schmidt, C. \& Urlaub, H. Combining cryo-electron microscopy (cryo-EM) and cross-linking mass spectrometry (CX-MS) for structural elucidation of large protein assemblies. Curr Opin Struct Biol 46, 157-168, doi:10.1016/j.sbi.2017.10.005 (2017).

Bruce, J. E. In vivo protein complex topologies: sights through a cross-linking lens. Proteomics 12, 15651575, doi:10.1002/pmic.201100516 (2012).

14 Young, M. M. et al. High throughput protein fold identification by using experimental constraints derived from intramolecular cross-links and mass spectrometry. Proc Natl Acad Sci U S A 97, 5802-5806, doi:10.1073/pnas.090099097 (2000).

Paramelle, D., Miralles, G., Subra, G. \& Martinez, J. Chemical cross-linkers for protein structure studies by mass spectrometry. Proteomics 13, 438-456, doi:10.1002/pmic.201200305 (2013).

Back, J. W., de Jong, L., Muijsers, A. O. \& de Koster, C. G. Chemical cross-linking and mass spectrometry for protein structural modeling. J Mol Biol 331, 303-313 (2003).

17 Yang, B. et al. Identification of cross-linked peptides from complex samples. Nat Methods 9, 904-906, doi:10.1038/nmeth.2099 (2012).

18 Gotze, M. et al. StavroX--a software for analyzing crosslinked products in protein interaction studies. J Am Soc Mass Spectrom 23, 76-87, doi:10.1007/s13361-011-0261-2 (2012). and identification of cross-linking sites using LC-MS/MS and the xQuest/xProphet software pipeline. Nat Protoc 9, 120-137, doi:10.1038/nprot.2013.168 (2014). 
(Palo Alto Calif) 8, 61-80, doi:10.1146/annurev-anchem-071114-040325 (2015).

21 Madler, S., Bich, C., Touboul, D. \& Zenobi, R. Chemical cross-linking with NHS esters: a systematic study on amino acid reactivities. J Mass Spectrom 44, 694-706, doi:10.1002/jms.1544 (2009).

22 Madler, S., Gschwind, S. \& Zenobi, R. Role of arginine in chemical cross-linking with Nhydroxysuccinimide esters. Anal Biochem 398, 123-125, doi:10.1016/j.ab.2009.11.020 (2010). Madler, S., Seitz, M., Robinson, J. \& Zenobi, R. Does chemical cross-linking with NHS esters reflect the chemical equilibrium of protein-protein noncovalent interactions in solution? J Am Soc Mass Spectrom 21, 1775-1783, doi:10.1016/j.jasms.2010.06.016 (2010).

24 Kalkhof, S. \& Sinz, A. Chances and pitfalls of chemical cross-linking with amine-reactive Nhydroxysuccinimide esters. Anal Bioanal Chem 392, 305-312 (2008). Leavell, M. D., Novak, P., Behrens, C. R., Schoeniger, J. S. \& Kruppa, G. H. Strategy for selective chemical cross-linking of tyrosine and lysine residues. J Am Soc Mass Spectrom 15, 1604-1611, doi:10.1016/j.jasms.2004.07.018 (2004).

26 Schein, C. H. Solubility as a function of protein structure and solvent components. Biotechnology $(N Y)$ 8, 308-317 (1990).

27 Itsik, P. e. et al. Proteomic signatures: Amino acid and oligopeptide compositions differentiate among phyla. Proteins: Structure, Function, and Bioinformatics 54, 20-40, doi:doi:10.1002/prot.10559 (2004). Wong, S. S., and Jameson, D. M. Chemistry of protein and nucleic acid cross-linking and conjugation. 2nd edn, (Taylor \& Francis/CRC Press, 2012).

Ding, Y. H. et al. Increasing the Depth of Mass-Spectrometry-Based Structural Analysis of Protein Complexes through the Use of Multiple Cross-Linkers. Anal Chem 88, 4461-4469, doi:10.1021/acs.analchem.6b00281 (2016).

Giese, S. H., Fischer, L. \& Rappsilber, J. A Study into the Collision-induced Dissociation (CID) Behavior of Cross-Linked Peptides. Mol Cell Proteomics 15, 1094-1104, doi:10.1074/mcp.M115.049296 (2016).

31 Kolbowski, L., Mendes, M. L. \& Rappsilber, J. Optimizing the Parameters Governing the Fragmentation of Cross-Linked Peptides in a Tribrid Mass Spectrometer. Anal Chem 89, 5311-5318, doi:10.1021/acs.analchem.6b04935 (2017).

32 Schneider, M., Belsom, A., Rappsilber, J. \& Brock, O. Blind testing of cross-linking/mass spectrometry hybrid methods in CASP11. Proteins 84 Suppl 1, 152-163, doi:10.1002/prot.25028 (2016).

33 Murakami, K. S. Structural biology of bacterial RNA polymerase. Biomolecules 5, 848-864, doi:10.3390/biom5020848 (2015).

34 Land, H. \& Humble, M. S. YASARA: A Tool to Obtain Structural Guidance in Biocatalytic Investigations. Methods Mol Biol 1685, 43-67, doi:10.1007/978-1-4939-7366-8_4 (2018).

35 Hofmann, T., Fischer, A. W., Meiler, J. \& Kalkhof, S. Protein structure prediction guided by crosslinking restraints--A systematic evaluation of the impact of the crosslinking spacer length. Methods 89, 79-90, doi:10.1016/j.ymeth.2015.05.014 (2015).

36 Merkley, E. D. et al. Distance restraints from crosslinking mass spectrometry: mining a molecular dynamics simulation database to evaluate lysine-lysine distances. Protein Sci 23, 747-759, doi:10.1002/pro.2458 (2014).

37 Kahraman, A., Malmstrom, L. \& Aebersold, R. Xwalk: computing and visualizing distances in crosslinking experiments. Bioinformatics 27, 2163-2164, doi:10.1093/bioinformatics/btr348 (2011).

38 Bullock, J. M. A., Sen, N., Thalassinos, K. \& Topf, M. Modeling Protein Complexes Using Restraints from Crosslinking Mass Spectrometry. Structure, doi:10.1016/j.str.2018.04.016 (2018).

39 Matthew Allen Bullock, J., Schwab, J., Thalassinos, K. \& Topf, M. The Importance of Non-accessible Crosslinks and Solvent Accessible Surface Distance in Modeling Proteins with Restraints From Crosslinking Mass Spectrometry. Mol Cell Proteomics 15, 2491-2500, doi:10.1074/mcp.M116.058560 (2016).

40 Borgstahl, G. E. How to use dynamic light scattering to improve the likelihood of growing macromolecular crystals. Methods Mol Biol 363, 109-129, doi:10.1007/978-1-59745-209-0_6 (2007). 
41 Green, N. S., Reisler, E. \& Houk, K. N. Quantitative evaluation of the lengths of homobifunctional protein cross-linking reagents used as molecular rulers. Protein Sci 10, 1293-1304, doi:10.1110/ps.51201 (2001).

42 Maciejewski, M., Barlow, P. N. \& Tjandra, N. Decoding the components of dynamics in three-domain proteins. J Comput Chem 35, 518-525, doi:10.1002/jcc.23510 (2014).

43 Garton, M., MacKinnon, S. S., Malevanets, A. \& Wodak, S. J. Interplay of self-association and conformational flexibility in regulating protein function. Philos Trans R Soc Lond B Biol Sci 373, doi:10.1098/rstb.2017.0190 (2018).

44 Fan, S. B. et al. Using pLink to Analyze Cross-Linked Peptides. Curr Protoc Bioinformatics 49, 821 21-19, doi:10.1002/0471250953.bi0821s49 (2015).

45 Bodnar, N. O. et al. Structure of the Cdc48 ATPase with its ubiquitin-binding cofactor Ufd1-Npl4. Nat Struct Mol Biol, doi:10.1038/s41594-018-0085-x (2018).

46 Potluri, S. et al. Geometric analysis of cross-linkability for protein fold discrimination. Pac Symp Biocomput, 447-458 (2004).

47 Vreven, T. et al. Integrating Cross-Linking Experiments with Ab Initio Protein-Protein Docking. J Mol Biol 430, 1814-1828, doi:10.1016/j.jmb.2018.04.010 (2018).

48 Schmidt, R. \& Sinz, A. Improved single-step enrichment methods of cross-linked products for protein structure analysis and protein interaction mapping. Anal Bioanal Chem 409, 2393-2400, doi:10.1007/s00216-017-0185-1 (2017). Leitner, A. et al. Expanding the chemical cross-linking toolbox by the use of multiple proteases and enrichment by size exclusion chromatography. Mol Cell Proteomics 11, M111 014126, doi:10.1074/mcp.M111.014126 (2012).

Chen, Z. A. et al. Architecture of the RNA polymerase II-TFIIF complex revealed by cross-linking and mass spectrometry. EMBO J 29, 717-726, doi:10.1038/emboj.2009.401 (2010).

\section{Figure legends}

Figure 1. Structures of the NHS-ester X-linkers DSM4, DSM6, and DSM8. A. Schematic of DSM6-derived X-link between 2 Lys residues (only CA-NZ chains are shown) defining CA-CA, CB-CB, NZ-NZ distances. B. Structural formula and the space-fill model of DSM4. C. Structural formula and the space-fill model of DSM6. D. Structural formula and the space-fill model of DSM8.

Figure 2. Molecular dynamics simulations of DSM4, DSM6, and DSM8 spacers. Conformational dynamics of NHS-ester X-linkers DSM4/6/8 reflected as temporal fluctuations of the interatomic distances between the first and the last carbon atoms of their respective spacer.

\section{Figure 3. Network view of 481 non-redundant inter-protein X-links between subunits of $E$. coli RNAP} subunits. Non-redundant inter-protein X-links aggregated from DSM4/6/8 datasets were mapped to the sequences of RNAP subunits. RpoA subunit present as a dimer in RNAP is shown here as a single copy due to the fact that XL-MS/MS does not permit unambiguous assignment of X-links within homo-oligomeric PPCs.

Figure 4. Flexible and rigid domains in the $E$. coli RNA polymerase. A. Structure of RNA polymerase core (4lk1), teal, with $\alpha$-helical hairpin (RpoB(936-1046), yellow), and unstructured loop (RpoC(36-103), purple) domains. B. $\alpha$-helical hairpin (RpoB(936-1046), gray ribbon) with X-linkable Lys residues 941, 988, 1007, and 1035 (ball and stick, gray, NZ atoms highlighted in blue). C. unstructured loop (RpoC(36-103), gray ribbon) with 
X-linkable Lys residues 50, 66, and 87 (ball and stick, gray, NZ atoms highlighted in blue).

Figure 5. Comparison of the conformational dynamics in rigid and flexible protein domains. A. Interatomic distances between backbone CA-CA (CA50-CA66, CA50-CA87) exhibit smaller amplitude than side chain NZ-NZ (NZ50-NZ66, NZ50-NZ66) in the unstructured loop domain (RpoC(36-103)). B. Interatomic distances between backbone CA-CA (CA941-CA1035, CA988-CA1007) exhibit smaller amplitude than side chain NZ-NZ (NZ941NZ1035, NZ988-NZ1007) in the rigid $\alpha$-helical hairpin (RpoB(936-1046) domain.

Figure 6. Comparison of the conformational dynamics of the NZ vs CA atoms in protein domains. A. NZ-NZ interatomic distances in the flexible domain (NZ50-NZ66, NZ50-NZ66) exhibit greater amplitude of molecular motion, compared to those in the rigid domain (NZ941-NZ1035, NZ988-NZ1007). B. CA-CA interatomic distances in the flexible domain (CA50-CA66, CA50-CA87) exhibit greater amplitude of molecular motion, compared to those in the rigid domain (CA941-CA1035, CA988-CA1007).

\section{Materials and Methods}

\section{Buffer components and consumables}

Buffer components for cross-linking and protein purification were BioUltra grade (Millipore Sigma). LCMS/MS was carried out with Thermo Scientific LC-MS grade reagents and solvents. Growth media components were from Thermo Fisher, protease inhibitor cocktail (ProBlock Gold Bacterial 2D) was from Gold Biotechnology. Low-binding pipet tips (Corning DeckWorks) and tubes (Protein LoBind, Eppendorf) were used throughout the experimental workflow.

\section{RNA polymerase expression and purification}

RNA polymerase core was expressed in $\mathrm{XJb}(\mathrm{DE} 3)$ E. coli cells (ZymoResearch) and purified as previously described $^{1}$, with additional polishing step: size exclusion chromatography on Superose Increase $610 / 300 \mathrm{GL}$ (GE) in X-linking buffer (50 mM HEPES (pH 7.5), 500 mM NaCl, $2 \mathrm{mM} \mathrm{MgSO}_{4}, 1 \mathrm{mM} \mathrm{DTT}$ ). Fractions containing pure RNAP core (analyzed by SDS-PAGE) and exhibiting polydispersity less than $10 \%$ and the molecular weight estimate of 385-390 kDa (389 kDa theoretical) in dynamic light scattering experiments ${ }^{2}$ (DynaPro Nanostar (Wyatt Technologies)) were pooled together and used in XL-MS experiments.

\section{XL-MS: X-linking, sample processing and analysis}

DSM4, DSM6, and DSM8 X-linkers (Proteochem) were dissolved in oxygen-depleted anhydrous DMSO (ZerO2, Millipore Sigma) at $100 \mathrm{mM}$ stock concentration and added to $100 \mu$ l of RNAP core solution (0.2 $\mathrm{mg} / \mathrm{ml}$ ) in X-linking buffer to final concentration of 250-500 $\mu \mathrm{M}$. X-linking was carried out for $45 \mathrm{~min}$ at $25^{\circ} \mathrm{C}$ in disposable cuvette (UVette, Eppendorf) with continuous monitoring of the polydispersity by dynamic light scattering to avoid aggregation of the sample (auto-attenuation of laser power, 10 acquisitions (5 sec each) per measurement). The reactions were quenched by addition of Tris- $\mathrm{HCl}(\mathrm{pH} \mathrm{7.5)}$ to a final concentration of 10 mM.

Samples were dialyzed against $100 \mathrm{mM}$ ammonium bicarbonate, reduced with $50 \mathrm{mM} \mathrm{TCEP}$ at $60^{\circ} \mathrm{C}$ for $10 \mathrm{~min}$ and alkylated with $50 \mathrm{mM}$ iodoacetamide in the dark for $60 \mathrm{~min}$ at $25^{\circ} \mathrm{C}$. Digestion was carried out at $37^{\circ} \mathrm{C}$ overnight with $0.5 \mu \mathrm{g}$ sequencing grade modified trypsin (Promega) in $100 \mathrm{mM}$ ammonium bicarbonate. The resulting peptides were passed though C18 Spin Tips (Thermo Scientific) before elution with $40 \mu \mathrm{L}$ of $80 \%$ acetonitrile (ACN) in $0.1 \%$ trifluoroacetic acid. Eluted peptides were dehydrated in vacuum and resuspended in $20 \mu \mathrm{L} 0.1 \%$ formic acid for MS analysis.

Peptides were analyzed in the Orbitrap Fusion Lumos mass spectrometer(Thermo Scientific) coupled to an EASY-nLC (Thermo Scientific) liquid chromatography system, with a $2 \mu \mathrm{m}, 500 \mathrm{~mm}$ EASY-Spray column. The peptides were eluted over a 120 -min linear gradient from $96 \%$ buffer A (water) to $40 \%$ buffer B (ACN), then 
continued to $98 \%$ buffer B over 20 min with a flow rate of $250 \mathrm{~nL} / \mathrm{min}$. Each full MS scan $(\mathrm{R}=60,000)$ was followed by 20 data-dependent MS2 $(R=15,000)$ with high-energy collisional dissociation (HCD) and an isolation window of $2.0 \mathrm{~m} / \mathrm{z}$. Normalized collision energy was set to 35. Precursors of charge state 4-6 were collected for MS2 scans; monoisotopic precursor selection was enabled and a dynamic exclusion window was set to $30.0 \mathrm{~s}$.

LC-MS/MS raw spectra were converted into mgf format using Proteome Discoverer (Thermo Fisher) and searched with pLink ${ }^{3}$. pLink $x$ link.ini file, containing X-linker search parameters, was amended to include DSM4=[K [K 110.0367110 .0367128 .0477128 .0477$, DSM6=[K [K 138.068138 .068156 .079 156.079, and DSM8=[K [K 166.0993166 .0993184 .1103 184.1103. X-linked peptide search space was defined by combining E. coli RpoA (P0A7Z4), RpoB (P0A8V2), RpoC (P0A8T7), and RpoZ (P0A800) sequences into RNAP.fasta file. Search parameters were defined in the pLink.ini file by setting the enzyme name to trypsin, maximal number of missed cleavages to 3, maximal e-value to 0.001 . Amino acid modifications were limited to 1 constant (Carbamidomethyl[C]), and 3 variable (Oxidation_M, Gln->pyro-Glu, and N-acetyl_Protein) ones. Samples of the ini and fasta files are included in the Supplement.

\section{Molecular dynamics simulations}

In order to estimate the dynamic range of DSM4/6/8 spacers in solution we performed molecular dynamic simulation of each reagent using YASARA Dynamics ${ }^{4}$ (YASARA Biosciences $\mathrm{GmbH}$ ). Starting structures in $p d b$ format were generated from DSM4/6/8 structural formula, MD simulation was carried out for $25 \mathrm{~ns}$ in explicit water with $0.9 \% \mathrm{NaCl}$, at $298 \mathrm{~K}$ in AMBER14 forcefield.

Starting $p d b$ coordinates for simulations of molecular motions of RpoB residues 936-1046 and RpoC residues 36-103 were extracted from 4lk1. Simulations were carried out for $20 \mathrm{~ns}$ in the same conditions as described above. For detailed information on the settings and outcome of the simulations see YASARA run and analysis macros included in the Supplement.

1 Svetlov, V. \& Artsimovitch, I. Purification of bacterial RNA polymerase: tools and protocols. Methods Mol Biol 1276, 13-29, doi:10.1007/978-1-4939-2392-2_2 (2015).

2 Borgstahl, G. E. How to use dynamic light scattering to improve the likelihood of growing macromolecular crystals. Methods Mol Biol 363, 109-129, doi:10.1007/978-1-59745-209-0_6 (2007).

3 Fan, S. B. et al. Using pLink to Analyze Cross-Linked Peptides. Curr Protoc Bioinformatics 49, 8 21 21-19, doi:10.1002/0471250953.bi0821s49 (2015).

4 Land, H. \& Humble, M. S. YASARA: A Tool to Obtain Structural Guidance in Biocatalytic Investigations. Methods Mol Biol 1685, 43-67, doi:10.1007/978-1-4939-7366-8_4 (2018). 